\title{
A NEW PURIFICATION METHOD FOR SINGLE BUNCH OPERATION AT THE PHOTON FACTORY STORAGE RING
}

\author{
K.Haga, T.Honda, T.Kasuga, T.Obina ${ }^{\dagger}$ and S.Sakanaka \\ KEK, 1-1 Oho, Tsukuba, Ibaraki 305-0801 Japan
}

\begin{abstract}
Recently, the requests for single bunch operation of the Photon Factory $2.5 \mathrm{GeV}$ electron storage ring (PF-ring) have gradually increased and the requirements for the impurity, that is the rate of electrons in undesirable buckets to those in the main bunch, becomes severe. A purification method making use of the current dependence of the betatron tune has been applied up to present. However, after the reconstruction of the PF-ring toward low emittance optics[1], it requires a mastery skill to keep an impurity better than $10^{-5}$ and moreover the transversal motion of the main bunch excited by the method could affect experiments of SR users. In order to overcome the situation and improve the purity, a new single-bunch purification method, that is essentially a "selective RF knock-out (RFKO)", has been developed. The system worked stably during the users time and the typical impurity of $0.6 \times 10^{-7}$ was achieved. Schematic description of the selective RFKO is shown in Fig.1, and related parameters of the PF-ring are shown in Table.1.
\end{abstract}

\section{INTRODUCTION}

Single bunch purity is very important for time resolved experiments such as Mössbauer spectroscopy, and the required impurity must be better than $1 \times 10^{-6}-10^{-7}$. The typical impurity after the short bunch injection stays in the order of $10^{-3}-10^{-4}$; consequently we must purity the spurious bunches in some method.

A transverse coupling impedance causes a shift in coherent betatron tune with the increase of the bunch current. Because the measured tune shift at the PF-ring was about $-2 \times 10^{-4} / \mathrm{mA}$, an RF knock-out (RFKO) tuned to the betatron frequency of the weak bunches can destroy the undesirable bunches with small currents but the main bunch with a larger current survives[2,3]. The frequency of RFKO and the aperture of a vertical scraper were adjusted preceded the users time, however, in some cases, it was difficult to keep the impurity better than $10^{-5}$ and we sometimes must re-configure such parameters. The difficulty is apparent after the reconstruction of the PFring because the non-linearity of the restoring forces becomes strong. Initial bunch current for SR users is about $60-70 \mathrm{~mA}$ and the corresponding vertical tune shift is about $10-15 \mathrm{kHz}$ while the spread in the coherent tune is

\footnotetext{
${ }^{\dagger}$ Email: obina@mail.kek.jp
}

larger than the tune shift. Namely, the RFKO signal affects not only the spurious bunches but also the main bunch itself.

We developed a new purification method which is very simple and straightforward. An RFKO signal is modulated by a rectangular pulse train with a repetition of the revolution frequency of the beam. Since the pulse train has gaps with a width of the bunch spacing (about $2 \mathrm{~ns}$ ), only a selected bunch passing through the RFKO kicker on the gap timing can survive. In order to avoid effects of non-linearity of the restoring forces acting on the beam, the excitation frequency is frequencymodulated.

Table 1: Main parameters of PF ring

\begin{tabular}{|l|c|c|}
\hline \multicolumn{1}{|c|}{ Parameters } & & unit \\
\hline Energy & 2.5 & $\mathrm{GeV}$ \\
\hline RF frequency & 500.1 & $\mathrm{MHz}$ \\
\hline momentum compaction & 0.061 & \\
\hline harmonic number & 312 & \\
\hline emittance & 36 & $\mathrm{~nm} \mathrm{rad}$ \\
\hline synchrotron frequency & 23 & $\mathrm{kHz}$ \\
\hline betatron tune $(\mathrm{vx} / \mathrm{vy})$ & $9.62 / 4.29$ & \\
\hline RF voltage & 1.7 & $\mathrm{MV}$ \\
\hline Radiation loss & 400 & $\mathrm{keV}$ \\
\hline
\end{tabular}

\section{STSTEM DESCRIPTION}

Figure 2 shows a block diagram of the system. A signal generator (MARCONI Inst., 2023) generates sinusoidal wave tuned to the vertical betatron resonant frequency, namely, $14 \times \mathrm{f}_{\text {rev }}+\mathrm{f}_{\beta_{\mathrm{y}}} \cong 22 \mathrm{MHz}$. Frequency modulation (maximum frequency deviation $100 \mathrm{kHz}$, modulation

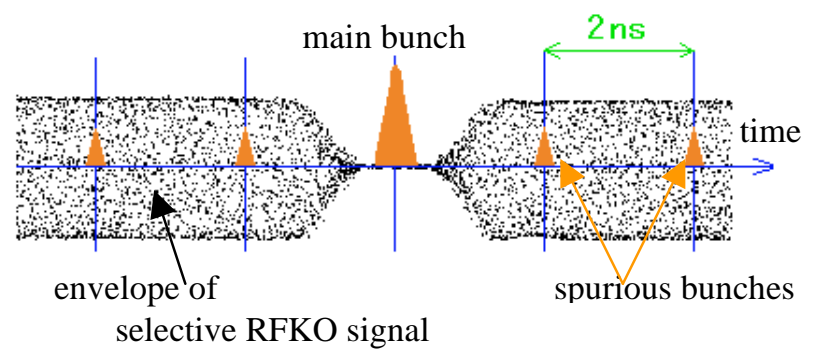

Figure 1: Schematic description of selective RFKO. 


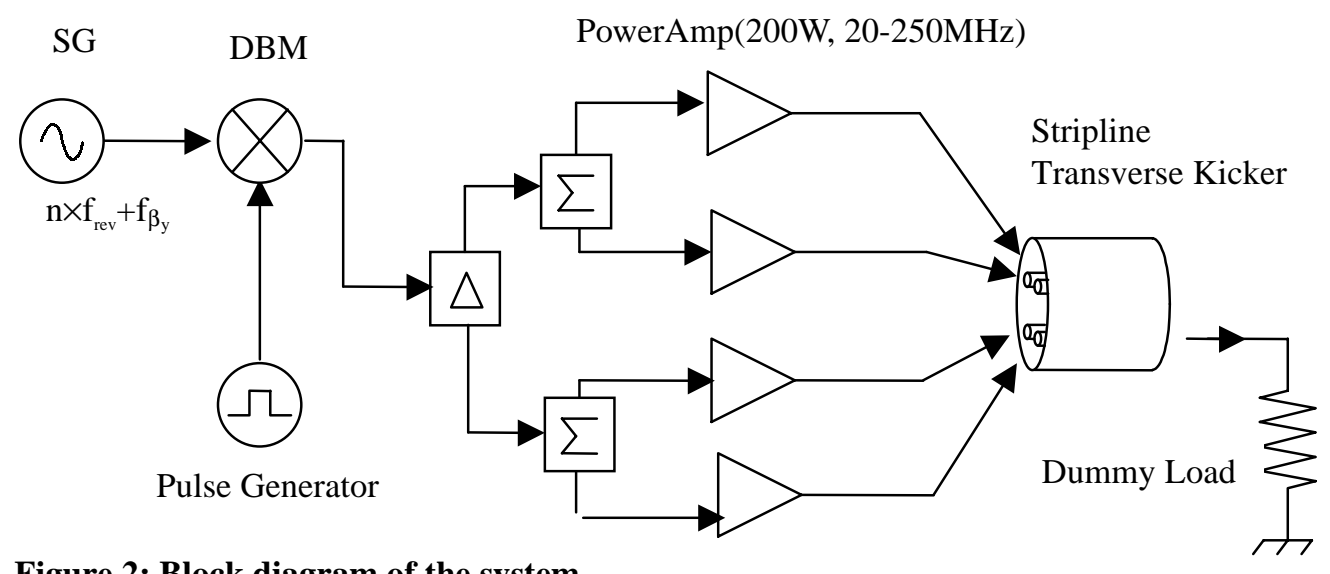

Figure 2: Block diagram of the system

frequency $\sim 4 \mathrm{~Hz}$ ) is applied in order to avoid effects due to non-linearity of the restoring forces acting on the beam. A double balanced mixer (DBM) is used to turn the KO signal off during the period ( $4 \mathrm{~ns})$ of passage of the main bunch. We tested several DBMs of several models in order to select a DBM which has excellent isolation between RF and LO port. The pulse generator HP8131A has a rise/fall time faster then the bunch spacing of $2 \mathrm{~ns}$, and an amplitude high enough to switch the DBM. The rise time of the pulse generator is faster than 200ps and the minimum pulse width is about 500ps with the amplitude of $\pm 5 \mathrm{~V}$. A timing signal synchronized to the bunch revolution generated by dividing the RF acceleration signal by 312 (harmonic number) is used as the trigger of the pulse generator.

The output of the DBM is splitted by a $180^{\circ}$ power splitter (mimi-curcuits), and splitted again with $0^{\circ}$ power splitters in order to produce the signal which kick the beam only in vertical direction. The wide-band signal processing is required to maintain the fast rise time of the signal.

Power amplifiers (R\&K A250-200-R) were previously used for the bunch-by-bunch feedback system during the high beam current study at the TRISTAN Accumulation Ring[4]. Its bandwidth and maximum power of $20-250 \mathrm{MHz}$ and $200 \mathrm{~W}$, respectively, are sufficient for our single bunch purification system. Four power amplifiers are connected to stripline electrodes of the transverse kicker. The output signals of the kicker electrodes are fed to the outside of the storage ring and used to adjust the timing of the pulse generator by observing the beam-induced signal and the RFKO signal simultaneously.

\section{RESULTS}

Figure 3 shows signals from button-type electrode when successive three bunches are injected (top) and removed except for the central bunch with the selective RFKO system (bottom). The pulse width was set to about 4 ns and the opening of the vertical scraper was $7 \mathrm{~mm}$ away from the centre of the beam.
A measurement of the bunch population is performed with a photon counting method using a PIN photodiode which has high sensitivity in X-ray region [57]. Figure 4 shows an example of photon counting measurement. The abscissa shows the time and the ordinate shows the counts of channels of MCA. Several spurious bunches are recognizable in every 2 ns before the purification(top). It is clearly seen that the spurious bunches are removed with the purification procedure. The measured impurity of the $1 \mathrm{st}$ bunch ( $2 \mathrm{~ns}$ behind the main bunch) was $8.3 \times 10^{-5}$ and $5.6 \times 10^{-7}$ before and after the purification, respectively. It took about 300 seconds to measure the impurity.

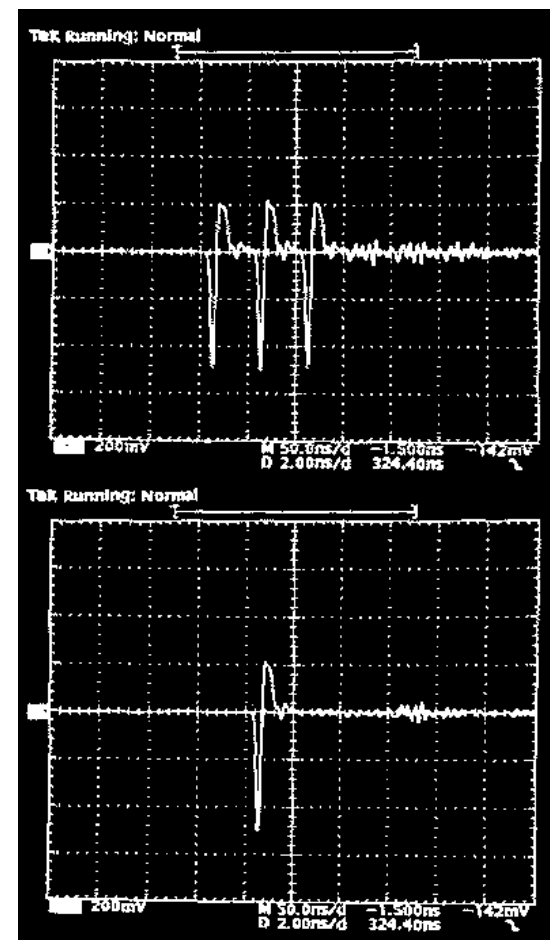

2ns/div

$200 \mathrm{mV} / \mathrm{div}$

Figure 3: Successive three bunches are injected (top) and knocked-out except for the central bunch (bottom). (2ns/div, 200mV/div) 

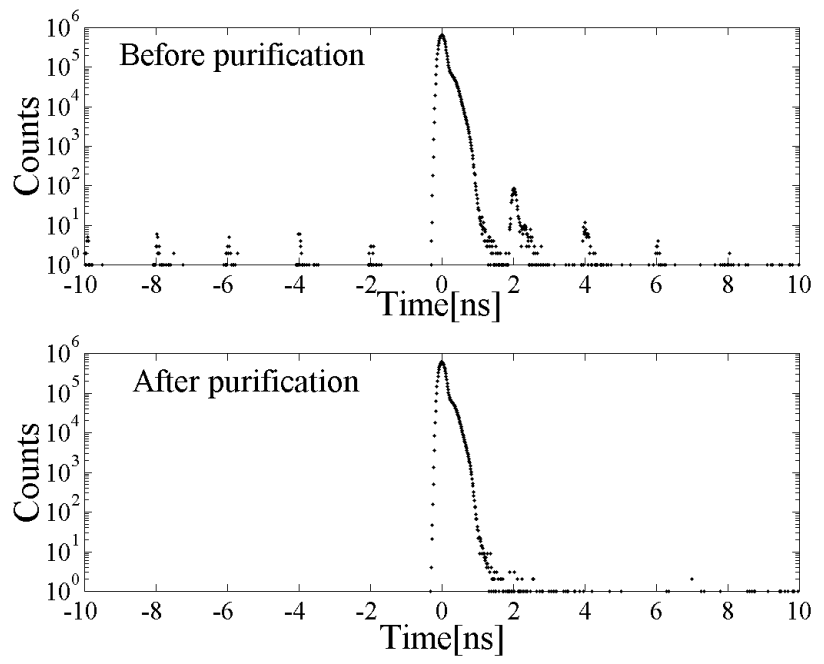

Figure 4: Bunch population measured with photon counting method before (top) and after (bottom) purification.

The rise time of the RFKO signal is fast enough, however, there are some ringing lasted for several nano seconds which can affect the main bunch. We decided the procedure of single bunch purification as shown below:

- Inject short bunches (about 70mA)

- Accelerates up to $3 \mathrm{GeV}$ to improve lifetime

- Increase H-V coupling to improve lifetime

- Remove leading bunches proceed the main bunch (for about $5 \mathrm{sec}$ )

- Remove trailing bunches after the main bunch (all time )

During the users time, we applied the purification system all the time because the impurity grows gradually without the purification. Some electrons thrown out from the main bunch are captured into the trailing buckets because there are openings of the separatrix due to the synchrotron radiation[8]. For example, an impurity of $4.6 \times 10^{-5}$ was measured one hour after the stop of purification. Because there are no apertures toward the foregoing buckets, the purification of them are performed only once after injection. The vertical scraper is not used in the routine operation because we could accomplish the enough impurity without it.

A typical result impurity measurement during the users time is shown in Fig.5. In this case, the impurity is better then $10^{-7}$ behind the main bunch. There is a small bunch before the main bunch, however, can be overcome by optimising the purification procedure.

The pulse generator and signal generator are remotely controlled by GPIB via LAN-HPIB gateway. HP-VEE is used for an operator interface for its easy and rapid development environment. It was not necessary to

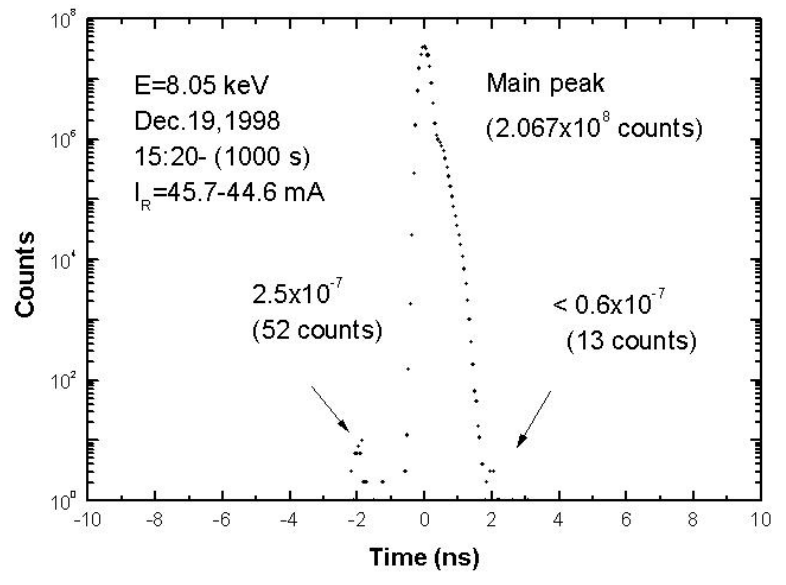

Figure 5: Typical impurity measurement during users time (courtesy of S. Kishimoto).

tune the system and to measure the impurity for every injection.

\section{SUMMARY}

We installed a new single bunch purification system at Photon Factory. An impurity of $2 \times 10^{-6} \sim 0.6 \times 10^{-7}$ was achieved during users time. The system works reliably and it becomes to an indispensable tool for single bunch operation. Several parameters (ex. FM modulation index, carrier frequency, clearing procedure) are not optimised yet. The impurity might be improved with the optimisation. We are installing the same system on the PF-AR (PF Advanced Ring).

The authors express their sincere thanks to Dr. S.Kishimoto for his great contribution for the X-ray photon counting system, and to Dr. Tobiyama who kindly provided the power amplifiers.

\section{REFERENCES}

[1] M. Katoh, et.al., "Operation of the Photon Factory with a High Brilliance Optics” EPAC'98 (1998)590

[2] Photon Factory Activity Report \#7 (1989) R-3

[3] Photon Factory Activity Report \#8 (1990) R-7

[4] M.Tobiyama and E. Kikutani, "Study of Bunch by Bunch Feedback System in TRISTAN-AR", Proc. Particle Acc. Conf. (1997)2335

[5] S. Kishimoto, Nucl. Instrum. and Methods, A309 (1991)603

[6] S. Kishimoto, Nucl. Instrum. and Methods, A397 (1997) 343

[7] S. Kishimoto, Rev. Sci. Instrum. 69(1998)384

[8] T.Obina, et.al, "Measurement of the longitudinal bunch structure in the Photon Factory positron storage ring with a photon counting system", Nucl. Instrum. and Methods A354(1995)204 